\title{
Bio-inspired flexible fiber brushes that keep liquids in a controlled manner by closing their ends
}

\author{
Tetsuya Yamamoto ${ }^{1,3}$, Qing'an Meng ${ }^{2}$, Qianbin Wang ${ }^{2}$, Huan Liu $^{2}$, Lei Jiang ${ }^{2,4}$ and Masao Doi ${ }^{1}$ \\ When brushes of flexible fibers are removed from liquid baths, these brushes sometimes show unwanted droplets at their ends, \\ depending on the length, rigidity and shape of their fibers. Capillary forces arising from the varying cross-sections of conical fiber \\ tips have been thought to eliminate the droplets. However, these forces may not operate with water, which fills the entire space \\ between the fibers of a brush. Here, we theoretically show that brushes eliminate unwanted droplets with a physical mechanism \\ that is significantly different from a single conical fiber by 'closing' their ends. We analyze the hydrostatics of water in a brush \\ when it is removed from a bath, and we identify the condition under which the end of the brush is closed, emphasizing the roles \\ played by the elastic deformation of the flexible fibers owing to interfacial forces. Moreover, this theory predicts that the volume \\ of water that is captured by brushes is a non-monotonic function of the length of their fibers because the fibers show excluded \\ volume interactions when their ends are 'closed'. This theory may guide the design of liquid-transfer devices that can retain \\ liquids in a controlled manner.
}

NPG Asia Materials (2016) 8, e241; doi:10.1038/am.2016.1; published online 5 February 2016

\section{INTRODUCTION}

Inspired by biological systems, liquid-transfer systems that are made of flexible fibers and/or plates have been designed in recent experiments. ${ }^{1-4}$ In many cases, these devices are dipped in liquid baths and are then removed to keep liquids in the devices, just like Chinese calligraphic brushes, which are traditional writing and painting tools in East Asia. The fibers of Chinese brushes are cylindrical, except for their conical ends. Without the conical fiber ends, Chinese brushes often show droplets at the ends of the brushes after they are removed from ink baths, where these remnant droplets may drop accidentally and leave stains on surfaces. ${ }^{1}$ The conical ends of the fibers thus have an important role in controlling liquids within Chinese brushes. Understanding the physical principle behind the hydrostatics of liquids in Chinese brushes may guide the design of liquid-transfer devices.

The stability of a liquid droplet on one fiber (or two) has been studied extensively. ${ }^{1,2,5-7}$ A droplet on a vertically aligned conical fiber moves to an equilibrium position, where gravitational forces (which tend to shift droplets downward) and capillary forces (which tend to shift droplets upward, where the curvature of the cross-section of the fiber is smaller) balance. ${ }^{1,5}$ This mechanical balance has been thought to be the physical mechanism involved in the ability of Chinese brushes with conical fiber ends to keep liquids without remnant droplets. ${ }^{1}$ However, in contrast to a droplet on a fiber, liquids in Chinese brushes fill the entire space between their fibers, and thus, the capillary forces arising from the varying cross-sections of the conical fiber ends do not operate because in such cases, the surface energy at the fiber-liquid interface is constant.

In general, when a Chinese brush is removed from a liquid bath, the liquid in the brush is still connected with the liquid in the bath via a capillary bridge because separating them requires a relatively large surface energy. When the capillary bridge shows instability, a part of the liquid in the bridge may stay at the end of the brush as a remnant droplet. The area of the end of the brush, including the cross-sections of the fiber ends and the space between the fibers, is a key parameter that determines the volume of the remnant droplet; the conical shapes of the fibers eliminate only the cross-sectional area of the fiber ends. Flexible fibers and plates at interfaces show elastic deformations owing to hydrostatic pressures and capillary forces. ${ }^{3,4,7-12}$ With sufficiently large hydrostatic pressures and/or capillary forces, the outmost fibers of a Chinese brush (which are at the interface between the ambient and the water) are bent toward the interior of the brush until the ends of these fibers touch at the end of the brush. Because the space between the fibers vanishes at the closed ends of brushes, closing the ends of brushes may eliminate the remnant droplets.

\footnotetext{
${ }^{1}$ Center of Soft Matter Physics and its Application, School of Chemistry and Environment, Beihang University, Beijing, China; ${ }^{2}$ Key Laboratory of Bio-Inspired Smart Interfacial Science and Technology of Ministry of Education, School of Chemistry and Environment, Beihang University, Beijing, China; ${ }^{3}$ National Composite Center, Nagoya University, Nagoya, Japan and ${ }^{4}$ Beijing National Laboratory for Molecular Science (BNLMS), Key Laboratory of Organic Solids, Institute of Chemistry, Chinese Academy of Science, Beijing, China

Correspondence: Dr T Yamamoto, National Composite Center, Nagoya University, Furocho, Chikusa-ku, 464-8603 Nagoya, Japan.

E-mail: tyamamoto@nuap.nagoya-u.ac.jp

or Professor H Liu, Key Laboratory of Bio-Inspired Smart Interfacial Science and Technology of Ministry of Education, School of Chemistry and Environment, Beihang University, Xueyuan Road 37, 100191 Beijing, China.

E-mail: liuh@buaa.edu.cn

Received 16 August 2015; revised 1 November 2015; accepted 19 November 2015
} 
With this mechanism, the volume of remnant droplets is limited by the elastic deformation of the outmost fibers of Chinese brushes. To highlight the roles played by the outmost fibers, we here use a model brush, where flexible fibers are end-grafted only to the edge of a diskshaped substrate. This structure is inspired by the fact that the seeds of dandelion show shapes that are analogous to Chinese calligraphic brushes when they are wetted by rain. ${ }^{3}$ The liquid-transfer systems of such structures have been demonstrated in recent research. ${ }^{3}$ Here we theoretically predict the shapes and volume of water that is captured by these model brushes when they are slowly removed from baths.

Our theory predicts that the ends of model brushes are closed before they are removed to the meniscus in cases in which the fibers of these brushes are longer than a critical length. The closed model brushes do not show remnant droplets at their ends because there is no surface-energy cost by separating the brushes from baths at their closed ends (where the area of the cross-section is zero). Moreover, this theory predicts that the volume of water that is captured by model brushes is a non-monotonic function of the length of fibers, where this function has one peak in the open state and another peak in the closed state. The second peak results from reaction forces owing to the excluded volume interactions between the fibers of closed brushes. Our results imply that closing the ends of Chinese brushes significantly affects the hydrostatic properties of these brushes. This theory may thus guide the design of liquid-transfer devices made of flexible fibers that can retain liquids in a controlled manner.

\section{MATERIALS AND METHODS}

\section{Model brush}

We treat a model brush, where flexible hydrophilic fibers are end-grafted to the edge of a disk-shaped substrate in the normal to the substrate surface with equal spacing; see Figure 1. When a model brush is dipped in a water bath and then slowly removed in the vertical direction, the region enclosed by the fibers of the brush is filled with water. ${ }^{3}$ Because the fibers of the model brush are

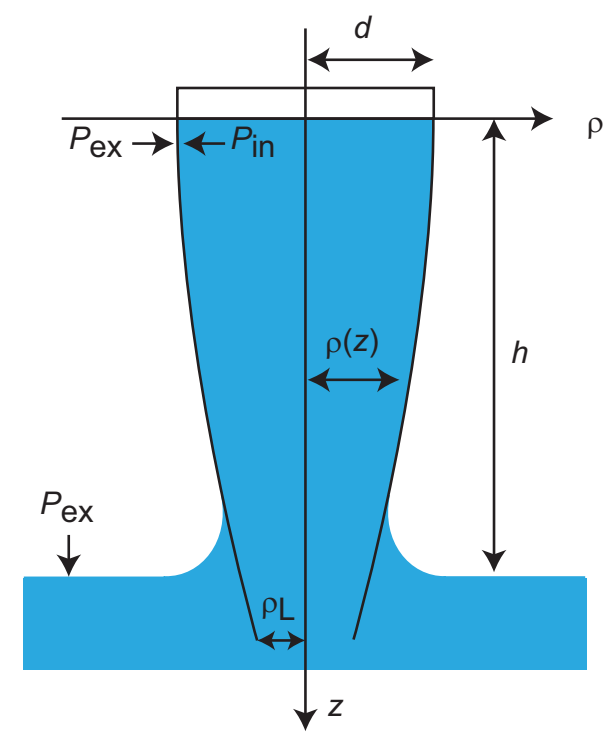

Figure 1 We treat a model brush in which flexible hydrophilic fibers are end-grafted to the edge of a disk-shaped substrate with equal spacing. When the model brush is dipped in a water bath and then slowly removed, water fills the region enclosed by the fibers. We use the axial coordinate system, where $z$ is the distance from the substrate, and $\rho(z)$ is the distance from the central axis. Height $h$ of the brushes is defined by the distance between the substrates and the level of the unperturbed surface of the water in the bath far away from the brush. hydrophilic, these fibers are right beneath the surface of the water to minimize the elastic energy of these fibers, and the entire length of the fibers is wetted; the fibers are bent following the shape of the ambient-water interface.

In a macroscopic treatment, the shape of a model brush that is composed of many fibers is represented by the axially symmetric envelop of the ambientwater interface; see Supplementary Information S1. With this treatment, the shapes of the fibers are represented by positional vectors that have the form $\vec{r}(z, \phi)=(\rho(z) \cos \phi, \rho(z) \sin \phi, z)$, where $\rho(z)$ is the distance between the ambient-water interface and the central axis of the brush, $z$ is the coordinate in the normal to the substrate surface and $\phi$ is the azimuthal angle; see Figure 1. Because hydrostatic pressures and capillary forces are applied to the fibers at the ambient-water interface (where these forces are opposed by the elastic forces of the fibers), the distance $\rho(z)$ is derived using the force-balance equation that has the form

$$
\Delta P-\rho_{0} g z+\frac{\gamma}{\rho(z)}+k_{\mathrm{fib}} \sigma(z) \rho^{\prime \prime \prime \prime}(z)=0,
$$

where the first and second terms are the difference between the ambient pressure and the hydrostatic pressures of water in the brush, the third term is capillary forces, and the fourth term is the elastic forces of fibers per unit area (see Supplementary Informations S1 and S2 for physical and formal derivations). Equation (1) is effective for the region of fibers already removed from baths, $z<h$. We used two approximations to derive equation (1): (i) gradient $\rho^{\prime}(z)(\sim d / L)$ is smaller than unity everywhere and (ii) curvature $\rho^{\prime \prime}(\mathrm{z})\left(\sim d / L^{2}\right)$ in the meridian direction is smaller than curvature $\rho^{-1}(z)(\sim 1 / d)$ in the radial direction; see Supplementary Information S2. $\Delta P\left(\equiv P_{\mathrm{ex}}-P_{\mathrm{in}}\right)$ is the difference between ambient pressure $P_{\mathrm{ex}}$ and hydrostatic pressures $P_{\text {in }}$ at $z=0$. When the end of the brush is in the bath, the pressure difference is $\rho_{0} g h$, where $\rho_{0}$ is the density of water, $g$ is the gravitational acceleration and $h$ is the brush height, which is defined by the distance between the brush substrate and the bath surface; see Figure 1. $\gamma$ is the surface tension. $k_{\mathrm{fib}}$ is the bending rigidity of each fiber and $\rho^{\prime \prime \prime \prime}(z)$ is the fourth derivative of $\rho(z)$ (with respect to the coordinate $z$ ). $\sigma(z)$ is the local number density of fibers (per unit area of the ambient-water interface) and has the form

$$
\sigma(z)=\frac{N_{\mathrm{fib}}}{2 \pi \rho(z)},
$$

where $N_{\text {fib }}$ is the number of fibers that are end-grafted to the substrate. The form of equation (1) is analogous to the shape equation of lipid membranes. ${ }^{13-15}$ The unique feature of equation (1) is that the (effective) local bending stiffness $k_{\mathrm{fib}} \sigma(z)$ per unit area increases as distance $\rho(z)$ between the surface and the central axis decreases; see equation (2). This is because the local number density of fibers increases as distance $\rho(z)$ decreases. The general solutions of equation (1) are expressed using hypergeometric functions; see Supplementary Information S3.

Forces are not applied to the region of fibers that are still in the bath, $z>h$; thus, the shape of this region is derived by the force-balance equation that has the form $\rho^{\prime \prime \prime \prime}(z)=0$. Distance $\rho(z)$ and its first, second and third derivatives are connected continuously at $z=h$. We neglect capillary rises along the sides of model brushes in the calculations of Figure $2 \mathrm{a}$ because we primarily treat situations in which model brushes are fully removed from bath surfaces, $h>L$.

We derive the shape, $\rho(z)$, of a model brush by enforcing four boundary conditions in the general solution of equation (1). Two of the boundary conditions are $\rho(0)=d$ and $\rho^{\prime}(0)=0$ for all cases (except for the calculations of Figure 4c; see the 'Comparison with experiments' section below). The other two boundary conditions depend on given situations and indeed define the states of model brushes: In the 'open' state, the radius of the end of the brush is not zero, and torques are not applied to the ends of the fibers, $\rho^{\prime \prime}(L)=0$. The radius $\rho(L)$ is determined by forces that are applied to the ends of the fibers. In cases where the ends of the fibers are in a bath, forces are not applied to the ends of the fibers, $\rho^{\prime \prime \prime}(L)=0$. Otherwise, traction forces owing to the surface tension of the free ambient-water interfaces (at the open ends or capillary bridges) are applied to the ends of the fibers, and thus, the boundary condition reads $k_{\mathrm{fib}} N_{\mathrm{fib}} \rho^{\prime \prime \prime}(L)-2 \pi \rho(L) \gamma \sin \theta_{c}=0$, where $\theta_{c}$ is the angle between the direction of the traction forces and the brush central axis. In the 'closed' (touch) state, the radius of the end of the brush is zero, $\rho(L)=0$, and torques are not applied to the ends of the fibers, $\rho^{\prime \prime}(L)=0$. In the 'closed' (parallel) 
a

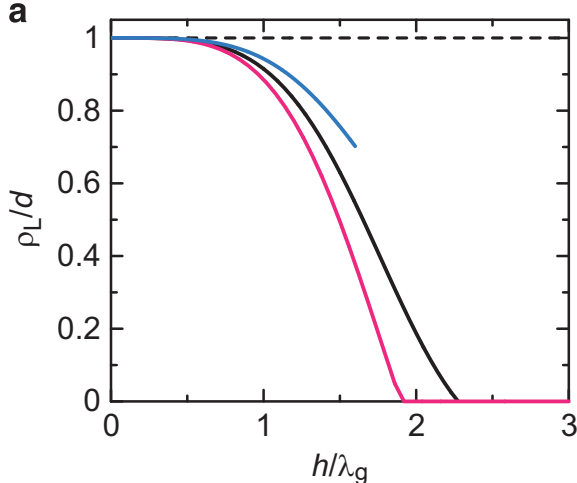

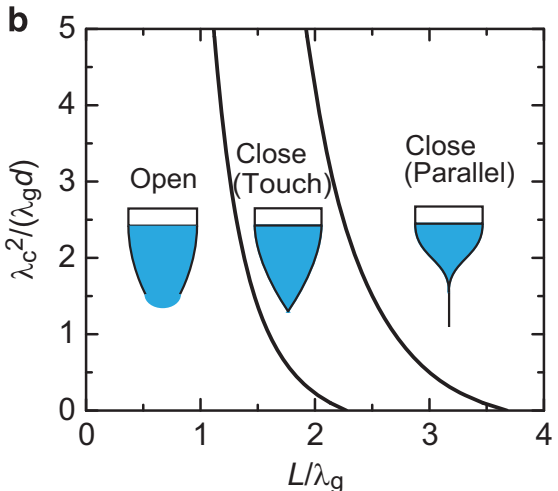

Figure 2 (a) Radius $\rho_{L}$ of the end of a model brush (rescaled by radius $d$ of the substrate) is shown as a function of height $h$ of the brush (rescaled by gravito-elastic length $\lambda_{g}$ ) for cases in which the pressure difference (between the ambient and the water in the brush) dominates capillary forces, $\lambda_{c}^{2} /\left(\lambda_{g} d\right)=0$. The values of rescaled fiber length $U \lambda_{g}$ used for the calculations are 1.6 (cyan), 2.275 (black) and 3.0 (magenta). (b) The diagram that shows the states ('open', 'close (touch)' and 'close (parallel)') of the model brushes as a function of length $L$ of the fibers (rescaled by the gravito-elastic length) and radius $d$ of the substrate (rescaled by length $\lambda_{c}^{2} / \lambda_{g}$ ). Closing length $L_{c l o}$ is the minimum length of fibers, where the ends of brushes are closed, and is a function of rescaled radius $d \lambda_{g} / \lambda_{c}^{2}$.

state, the radius of the end of the brush is zero, $\rho\left(z_{\mathrm{con}}\right)=0$, and the tangent of the ends of the fibers is parallel to the central axis, $\rho^{\prime}\left(z_{\text {con }}\right)=0$, where $z_{\text {con }}$ is the minimum of the $z$-coordinate of the region, at which distance $\rho(z)$ is zero. Boundary condition $\rho(L)=0$ implies that reaction forces $k_{\mathrm{fib}} \rho$ "' $(L)$ owing to the excluded volume interactions between fibers are not zero in the closed state. ${ }^{16}$

\section{Capillary bridges}

The shape of the ambient-water interface of a capillary bridge is represented by a positional vector that has the form $\vec{r}_{c}(z, \phi)=\left(\rho_{c}(z) \cos \phi, \rho_{c}(z) \sin \phi, z\right)$, where $\rho_{c}(z)$ is the distance between the central axis and the ambient-water interface. Distance $\rho_{c}(z)$ is derived by using the Laplace equation that has the form

$$
\rho_{0} g(h-z)+\gamma\left[\frac{1}{\rho_{c}(z)} \frac{1}{\sqrt{1+\rho_{c}^{\prime 2}(z)}}-\frac{\rho_{c}^{\prime \prime}(z)}{\left(1+\rho_{c}^{\prime 2}(z)\right)^{3 / 2}}\right]=0,
$$

The first term is the difference between the ambient pressure and hydrostatic pressures, and the second term is capillary forces. We derive the solution to equation (3) by enforcing two boundary conditions: (i) the height of the capillary bridge asymptotically approaches the unperturbed surface far away from the brush, $\rho_{c}(h) \rightarrow \infty$ and (ii) the surface of the capillary bridge is continuously connected to the end of the brush, $\rho_{c}(L)=\rho_{L}$, where $\rho_{L}(\equiv \rho(L))$ is the radius of the end of the brush. The asymptotic solutions to equation (3) for $\rho_{L}<\lambda_{c}$ and $\rho_{L}>\lambda_{c}$ are shown in Supplementary Information S5. We use the solutions for $\rho_{L}<\lambda_{c}$ in the rest of this paper. Radius $\rho_{L}$ at the end of a model brush decreases as height $h$ of the brush increases because hydrostatic pressures and/or capillary forces bend the brush fibers toward the interior of the brush; see equation (1). Moreover, end radius $\rho_{L}$ depends on traction forces that are generated by the surface tension because of the free ambient-water interfaces of the capillary bridge, where these traction forces depend on the shapes of the capillary bridge (via angle $\theta_{\dot{c}}$, see the 'Model brush' section and Supplementary Information S5 and Supplementary Figure S3). The solution to equations (1) and (3) exists only for brush heights $h$ that are smaller than a critical height $h_{\mathrm{cr}}$. This implies that the capillary bridge shows instability at the critical height and ruptures; see Supplementary Information S5.

When the capillary bridge ruptures, some of the water in the capillary bridge returns to the bath, and the rest is included in the brush. In general, the volume of water that is included in the brush depends on the dynamics of water in the capillary bridge during the rupture. We here use a simple approximate treatment, where the water above the minimal cross-section of the capillary bridge at critical height $h_{\mathrm{cr}}$ is included in the brush, and the rest of water returns to baths. This treatment is rationalized by the fact that separating the water in the brush and the water in the bath at the minimal cross-section costs minimal surface energy. Volume $V$ of water that is captured by a model brush is thus the sum of the two volumes, $V=V_{\mathrm{drp}}+V_{\mathrm{fib}}$ : (i) volume
$V_{\mathrm{drp}}=\int_{L}^{z_{\min }} d z \pi \rho_{c}^{2}(z)$ of water that is added from capillary bridges and (ii) volume $V_{\text {fib }}=\int_{0}^{L} d z \pi \rho^{2}(z)$ of water in the region that is enclosed by the fibers of the brush, where $\rho_{c}(z)$ and $\rho(z)$ for the critical height should be used to calculate the volume, and $z_{\min }$ is the $z$-coordinate of the minimal cross-section of the capillary bridge; see Supplementary Information S5.

\section{Model brush that is separated from baths}

The shape of a model brush after it is separated from a bath is calculated by treating $\Delta P$ in equation (1) as a Lagrange multiplier that fixes the volume of water in the brush to $V$; see also the 'Capillary bridge' section. In the open state, the shape of the ambient-water interface at the open end of a model brush is represented by positional vector $\vec{r}_{\text {opn }}(\rho, \phi)=\left(\rho \cos \phi, \rho \sin \phi, L-h_{\text {opn }}(\rho)\right)$. The height, $h_{\mathrm{opn}}(\rho)$, of the interface is derived using a force-balance equation that has an approximate form

$$
\Delta P+\rho_{0} g\left(h_{\mathrm{opn}}(\rho)-L\right)+\gamma \frac{1}{\rho} \frac{\partial}{\partial \rho}\left(\rho \frac{\partial}{\partial \rho} h_{\mathrm{opn}}(\rho)\right)=0 .
$$

We enforce two boundary conditions in the solution to equation (4): i) Height $h_{\text {opn }}(\rho)$ does not diverge at $\rho=0$ and (ii) the interface intersects with the ends of the fibers, $h_{\mathrm{opn}}\left(\rho_{L}\right)=0$. The form of the solution to equation (4) is shown in Supplementary Information S7. Equation (4) is effective for cases in which gradient $\left|h_{\mathrm{opn}}{ }^{\prime}(\rho)\right|$ is smaller than unity everywhere in the open ends. We thus use approximation $\theta_{c} \approx \pi / 2$ to derive the shape of a model brush in the open condition; see the 'Model brush' section.

\section{Comparison with experiments}

We rescaled the experimental results published in the study by Meng et al. ${ }^{3}$ to derive Figure $4 \mathrm{c}$; see reference 3 for the details of the experiments. Briefly stated, model brushes were prepared by end-grafting glass fibers (diameter $=\sim 20 \mu \mathrm{m}$, bending rigidity $=5.6 \times 10^{-10} \mathrm{Nm}^{-2}$, contact angle $\left.=63.2^{\circ} \pm 1.2^{\circ}\right)$ to the edge of a polymer rod in the radial direction with equal spacing (the contact angle was measured using DataPhysics C20). The brushes were dipped in water baths at a speed of $1.7 \mathrm{~mm} \mathrm{~s}^{-1}$ until they were entirely wet. Then, the brushes were removed from the baths at the same speed. The weight of the captured water was measured.

Equation (1) is not effective for the region of radially end-grafted fibers $\left(\rho^{\prime}(0) \rightarrow \infty\right)$ at the proximity of the substrate, where the gradient $\rho^{\prime}(z)$ is larger than unity. As an approximate treatment, we here take into account only the region in which gradient $\rho^{\prime}(z)$ is smaller than unity because this region mostly accounts for the volume of water in the brushes. ${ }^{3}$ We thus derive the solution to equation (1) using the boundary conditions, $\rho(0)=d$ and $\rho^{\prime}(0) \simeq 1$, to compare with the experiments; see Figure $4 c$. 


\section{Model experiments using dandelion seeds}

A dandelion seed was freshly removed from the grassland of Beihang University $\left(39.98^{\circ} \mathrm{N}, 116.34^{\circ} \mathrm{E}\right)$. The number of pappi (fibers) of the seed was $\sim 105$, the radius of the substrate was $\sim 350 \mu \mathrm{m}$ and the angle between the grafted end of fibers and the central axis was $84^{\circ}$. A dandelion seed was dipped in a water bath and then removed in the vertical direction with a speed of $100 \mu \mathrm{m} \mathrm{s}^{-1}$ using a motorized stage (Dataphysics DCTA 11, Filderstadt, Germany). This process was recorded using a video camera (WV-CP280/CH, Panasonic, Osaka, Japan). The same experiment was performed using the same dandelion seed, but the fibers were cut by $\sim 1 \mathrm{~mm}$. The results are shown in Supplementary Figure S4.

\section{RESULTS}

\section{Phase diagram of model brush}

To identify the conditions under which the ends of the brushes are closed, we first analyze the shapes of model brushes when they are slowly (quasi-statically) removed from water baths in the vertical direction. The hydrostatic pressure of water in brushes is smaller than the ambient pressure owing to gravitational forces that are applied to the water in brushes; see Figure 1. For cases in which the pressure difference dominates capillary forces, the only relevant length scale is the gravito-elastic length, which has the form

$$
\lambda_{g} \equiv\left(\frac{k_{\mathrm{fib}} N_{\mathrm{fib}}}{2 \pi \rho_{0} g}\right)^{1 / 5}
$$

The bending rigidity, $k_{\mathrm{fib}}$, and number, $N_{\mathrm{fib}}$, of fibers is included in our theory only via the gravito-elastic length. When the height $h$ of a model brush is smaller than the gravito-elastic length, the pressure difference is not large enough to bend the fibers of the brush; see Figure $2 \mathrm{a}$. In contrast, when brush height $h$ is larger than the gravitoelastic length, the fibers of the brush are gradually bent toward the interior of the brush while the brush is removed from the bath; radius $\rho_{L}$ of the end of a model brush decreases as height $h$ increases. ${ }^{4}$

Our theory predicts that the end of a model brush is closed before the brush end is brought up to the level of the unperturbed surface of the bath (far away from the brush) for cases in which the fibers of the brush are longer than critical length $L_{\text {clo }}$ (which we call the 'closing' length); see the magenta curve in Figure $2 \mathrm{a}$. In contrast, when the fibers of a brush are shorter than the closing length, the end of the brush is still open when the brush end is brought to the bath surface; see the cyan curve in Figure 2a. Indeed, with a general argument, one can show that the state of a model brush (whether its end is closed or open) is determined by whether its fibers are longer or shorter than the closing length; see Supplementary Information S6. One can thus draw a phase diagram with respect to the states of model brushes as a function of the (rescaled) fiber length and the (rescaled) radius of brush substrates; see Figure 2b. a

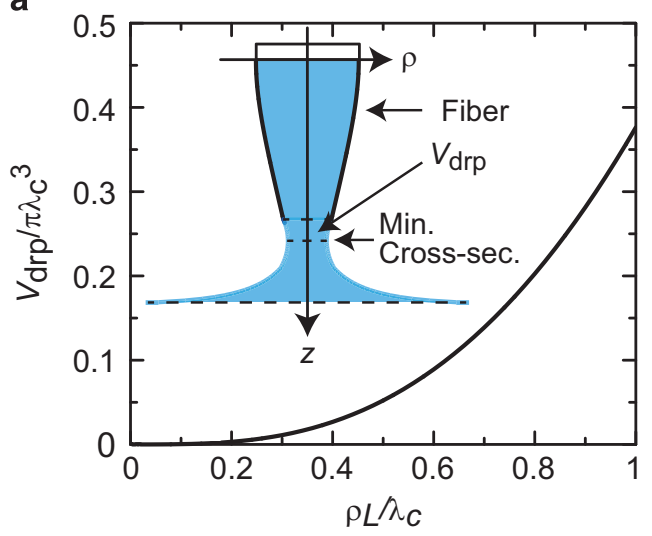

b

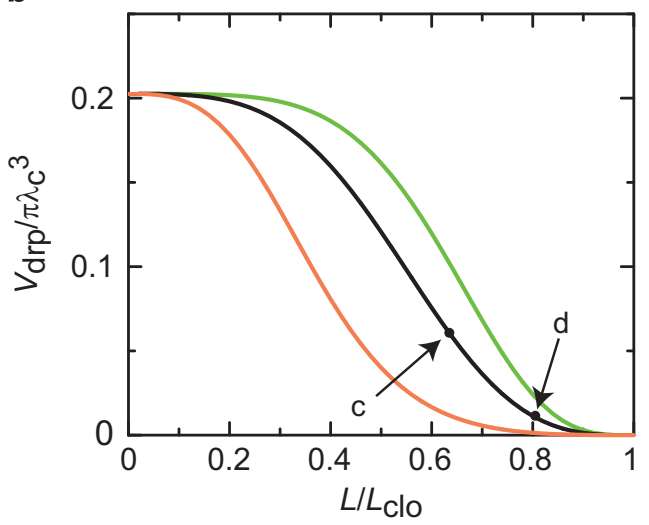

C

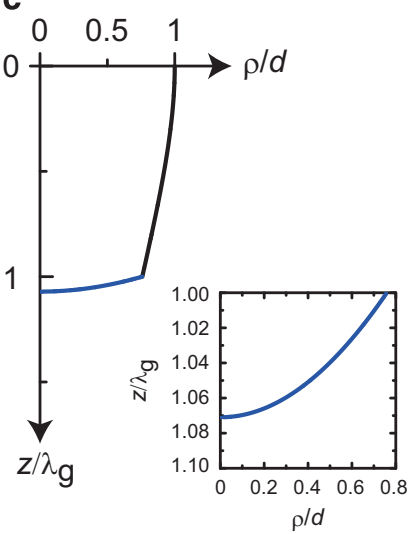

d

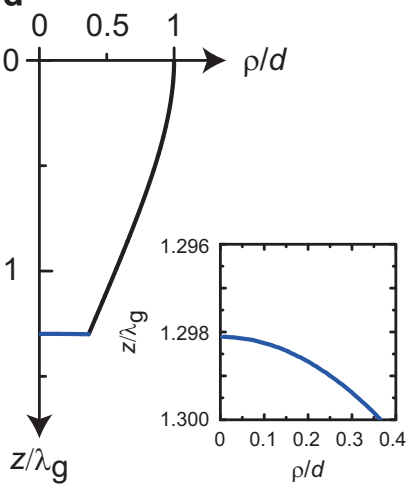

Figure 3 (a) Volume $V_{\text {drp }}$ of a remnant droplet, which stays at the end of a model brush owing to the rupture of capillary bridges (see inset), is shown as a function of radius $\rho_{L}$ of the end of the brush. Volume $V_{\text {drp }}$ and radius $\rho_{L}$ are rescaled using capillary length $\lambda_{c}$. (b) Volume $V_{\text {drp }}$ of water, which is added to model brushes from the capillary bridges after the rupture of capillary bridges, is shown as a function of length $L$ of flexible fibers. The volume of water is rescaled by capillary length $\lambda_{c}$ and fiber length $L$ is rescaled by closing length $L_{c l o}$; see Figure 2 . The values of ratio $\lambda_{d} d \lambda_{g}$ of length scales that are used for the calculations are 0.08 (light green), 0.8 (black) and 4.0 (orange), where $\lambda_{g}$ is the gravito-elastic length. (c, d) The shapes of the fibers (black) of model brushes and the ambient-water interface (blue) at their open end, after the brushes are completely removed from baths, are shown for cases in which (rescaled) fiber length $L / \lambda_{g}$ is 1.0 (c) and 1.3 (d). The insets show the shapes of the ambient-water interfaces. The value of ratio $\lambda_{d} \lambda_{g}$ used for the calculations is 0.8 for both $\mathbf{c}, \mathbf{d}\left(L_{c \mid l} / \lambda_{g} \simeq 1.6\right)$. The value of radius $d / \lambda_{c}$ of the substrate is fixed at 0.8 for the calculations of $\mathbf{b}, \mathbf{c}$ and $\mathbf{d}$. 
For cases in which radius $d$ of the substrates is larger than threshold value $d_{\mathrm{th}}\left(\sim \lambda_{c}^{2} / \lambda_{g}\right)$, the pressure difference dominates capillary orces (hydrostatic regime), where $\lambda_{c}\left(\equiv \sqrt{\gamma / \rho_{0} g}\right)$ is the capillary length. ${ }^{6}$ In this regime, closing length $L_{\text {clo }}$ scales linearly with the gravito-elastic length $\lambda_{g}$; see Figure $2 \mathrm{~b}$ and Supplementary Figure S2. This implies that in contrast to brushes of elastic plates (see Supplementary Information S8), closing length $L_{\text {clo }}$ is independent of radius $d$ of the substrate, although the distances by which the ends of the fibers must be displaced to close the ends of the brushes increase as radius $d$ increases. This is because the local bending stiffness of the surfaces of the model brushes decreases as radius $d$ of their substrates increases; see equation (2). For cases in which radius $d$ is smaller than threshold radius $d_{\text {th }}$, capillary forces dominate the pressure difference (capillary regime). In this regime, the closing length of fibers scales as $L_{\mathrm{clo}} \sim \lambda_{g_{g}}^{5 / 4} d^{1 / 4} / \lambda_{c}^{1 / 2}$; see Figure $2 \mathrm{~b}$ and Supplementary Figure S2. A scaling argument that shows the above scaling relationship of the closing length is shown in Supplementary Information S4.

Tangent vectors at the ends of the fibers of model brushes are gradually redirected toward the central axes as the height of the brushes increases. For cases in which the fibers of the brush are longer than a second critical length, the tangent vectors become parallel to the central axes before the ends of the brushes reach the meniscus; see Figure 2b. Because water in these parallel brushes is not stable, we here primarily treat model brushes that are composed of shorter fibers; see also Supplementary Information S9.

\section{Remnant droplets that stay at the ends of brushes}

For the simplest cases, in which a model brush is composed of very stiff fibers, $L \ll \lambda_{g}$, the radius of the end of such a stiff brush is approximately constant, $\rho_{L} \approx d$; see Figure $2 \mathrm{a}$. When the brush is brought up to a height higher than the level of the bath surface, $h>L$, the end of the brush is still connected with water in the bath via capillary bridges because separating them requires relatively large surface energy (see the inset of Figure 3a). This situation is analogous to cases in which a hard $\operatorname{rod},{ }^{17}$ a hard $\operatorname{ring}^{6,18}$ or a hard plate $^{19}$ is removed from a bath. When the stiff brush is brought up to critical height $h_{\mathrm{cr}}$, the capillary bridges show instability and rupture; see also Figure 3a and Supplementary Information S5. For simplicity, we here use an approximate treatment whereby at the rupture, the water below the minimal cross-section of the capillary bridge returns to the bath, and the rest of the water stays at the end of the brush as a (remnant) droplet; see the inset of Figure 3a. This treatment is rationalized by the fact that the surface-energy cost is minimal when capillary bridges are separated at the minimal cross-section (see also the discussion below). The volume of the remnant droplet decreases as radius $\rho_{L}$ of the end of the brush decreases and tends to zero for $\rho_{L} \rightarrow 0$; see Figure $3 \mathrm{a}$. Radius $\rho_{L}$ of the end of a model brush at the critical height is thus a key parameter that determines the volume of the remnant droplet that remains at the end of the brush.

Radius $\rho_{L}$ of the end of a model brush that is composed of flexible fibers decreases as height $h$ of the brush from the surface of a bath increases; see Figure 2a. For cases in which the fibers of the brush are longer than the closing length, radius $\rho_{L}$ of the brush end is zero when the brush end is brought up to the level of the bath surface, $h=L$; there is no surface-energy cost of separating the brush from the bath, where the area of the cross-section is zero. The brush is thus separated from the bath without showing a capillary bridge. No droplet remains at the end of the closed brush; see $L=L_{\text {clo }}$ in Figure $3 \mathrm{~b}$. In contrast, for cases in which the fibers of the brush are shorter than the closing length, radius $\rho_{L}$ at the brush ends is larger than zero when the brush end is brought up to the bath surface. The brush is thus connected with the bath via a capillary bridge when the brush height is smaller than a critical height, $h<h_{\mathrm{cr}}$. In contrast to the cases of stiff brushes, end radius $\rho_{L}$ of a flexible brush continues to decrease as the height of the brush increases owing to the gravitational forces that are applied to the water in the capillary bridge. Nevertheless, end radius $\rho_{L}$ never becomes zero before the brush is brought up to the critical height; see Supplementary Information S6. When the capillary bridge ruptures, there is always a finite volume of water that is included in the brush; see $L<L_{\text {clo }}$ in Figure $3 \mathrm{~b}$. In some cases, the water stays at the end of the brush as a remnant droplet; see Figure 3c. This finding is in agreement with experiments on an analogous system; see also Supplementary Figure S4. The volume of water that is added to the model brush from the capillary bridge increases as the bending rigidity $k_{\mathrm{fib}}\left(\sim \lambda_{g}^{5}\right)$ of their fibers increases; see Figure $3 b$.

When a model brush is separated from a bath, the model brush attains a new mechanical balance, with a volume $V_{\text {drp }}$ of water that is added from the capillary bridges. For cases in which the brush is composed of relatively stiff fibers, $L<\lambda_{g}$, the added water stays at the end of the brush as a remnant droplet; see Figure $3 \mathrm{c}$. In contrast, for cases in which the brush is composed of more flexible fibers, $L \sim L_{\text {clo }}$ (but is still shorter than closing length $L_{\text {clo }}$ ), the water from a capillary bridge is sucked into the interior of the brush. This occurs because the hydrostatic pressures of water in the brush slightly increase when the capillary bridge ruptures owing to the release of gravitational forces of water that returns to the bath. The hydrostatic pressures slightly push their fibers toward the exterior of the brush, and the free surface at the open end of the brush shows a concave (but almost flat) shape; see Figure 3d. However, in more general cases, in which a model brush is removed with a finite speed, the volume of water that is included in the brush from the capillary bridge may be sensitive to the dynamics of water during the rupture of the capillary bridge; in some cases, the volume of added water may be large enough to stay as a droplet. We thus argue that closing the ends of brushes more robustly eliminates remnant droplets because capillary bridges are not stabilized.

\section{Volume of water in brushes}

The volume of water that is captured by a model brush from a bath is a hydrostatic quantity that characterizes the functions of the brush. Our theory predicts that the volume of water that is captured by model brushes is a non-monotonic function of the length of their fibers. The volume shows a peak in the open state and another peak in the closed state; see Figure 4a. Two competing effects account for the peak in the open state, $L<L_{\text {clo: }}$ First, the height of the region that is enclosed by the fibers of model brushes increases as the fiber length increases; the volume of water that is captured by such brushes increases as length $L$ of the fibers increases. This geometrical effect dominates in cases where brush fibers are relatively short. Second, the pressure difference, $\Delta P\left(\sim \rho_{0} g L\right)$, which bends the fibers of brushes toward the interior, increases as length $L$ of their fibers increases; see also Figures $3 \mathrm{c}$ and $\mathrm{d}$. The volume of water that is captured by such brushes decreases as the length of the fibers increases. This mechanical effect dominates for brushes that have relatively long fibers. The volume of remnant droplets makes only minor contributions to the total volume unless the fibers of brushes are too short, $L<d$.

In the closed state, reaction forces that oppose the pressure difference are applied to the ends of the fibers of brushes owing to 
a

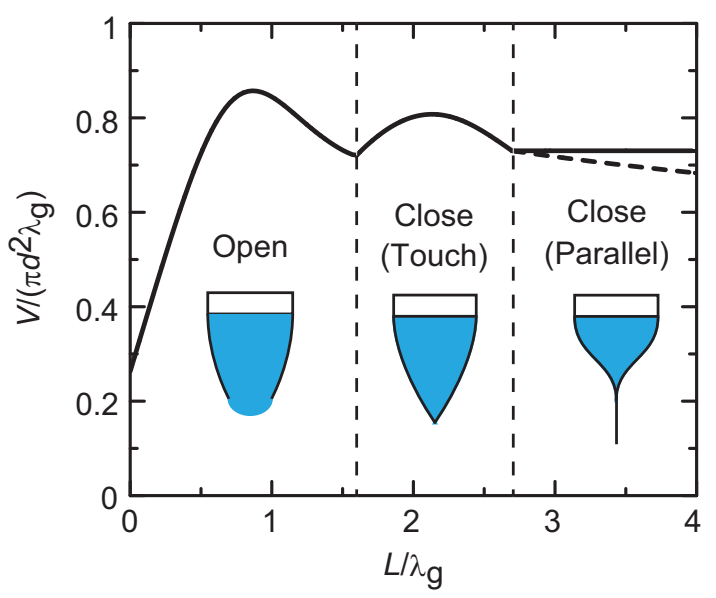

b

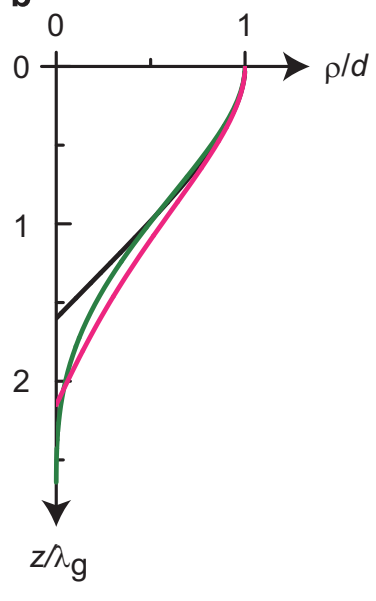

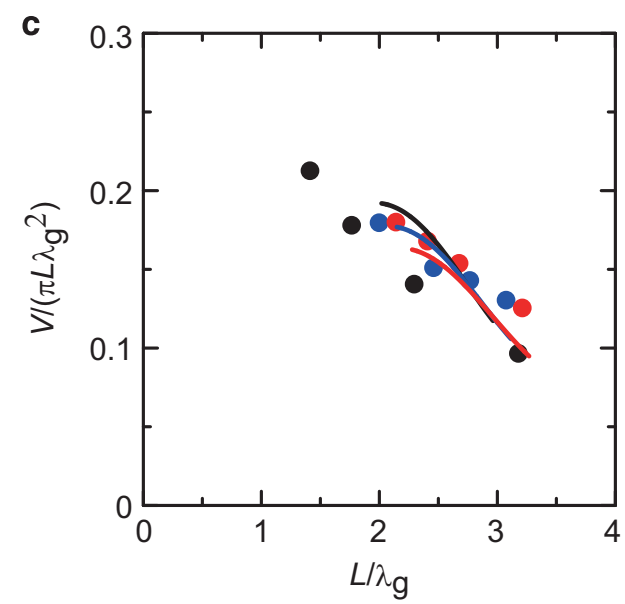

Figure 4 (a) Volume $V$ of water (rescaled by $\pi d^{2} \lambda_{g}$ ) that is captured by model brushes from baths is shown as a function of length $L$ of the fibers of these brushes (rescaled by $\lambda_{g}$ ), where $\lambda_{g}$ is the gravito-elastic length, and $d$ is the radius of the brush substrate. The values of ratio $\lambda_{d} \lambda_{g}$ and rescaled radius $d / \lambda_{c}$ that are used for the calculation are both 0.8 ( $\lambda_{c}$ is the capillary length). In the parallel state, the solid and broken curves show upper and lower bounds of the volume of water that is captured by brushes, respectively. (b) The shapes of model brushes are shown for cases in which the values of rescaled fiber length $L \lambda_{g}$ are 1.6 (black), 2.15 (magenta) and 2.7 (green). The value of rescaled radius $\lambda_{g} d / \lambda_{c}^{2}$ that is used for the calculations is 1.0 . (c) Volumes $V$ of water (rescaled by $\pi L \lambda_{g}^{2}$ ) that is captured by model brushes that are composed of 20 (black), 40 (blue) and 80 (red) fibers are shown as functions of rescaled fiber length $L$ (rescaled by the gravito-elastic length $\lambda_{g}$ ). The dots are extracted from recent measurements, ${ }^{3}$ and the solid lines are predicted by our theory. Bending rigidity $k_{\mathrm{fib}}$ of fibers that are used for the rescaling and the calculations is $5.6 \times 10^{-10} \mathrm{~N} \mathrm{~m}^{-2}$. See also Supplementary Information S9 for the closed (parallel) regime.

the excluded volume interactions between these fibers. For cases in which the fibers of brushes are relatively short, $L \sim L_{\text {clo }}$ (but are still longer than closing length $L_{\mathrm{clo}}$ ), the pressure difference is not large enough to bend the fibers, owing to the reaction forces. Thus, the ends of the fibers of the brushes just shift to lower positions as length $L$ of the fibers increases; see the magenta curve in Figure $4 \mathrm{~b}$. The volume of water that is captured by brushes thus increases as length $L$ of their fibers increases. For cases in which the fibers of brushes are relatively long, the pressure difference can be large enough to bend the fibers toward the interior of the brushes; see the green curve in Figure $4 \mathrm{~b}$. Because the pressure difference increases with increasing fiber length, $\Delta P=\rho_{0} g L$, the volume of water that is captured by brushes decreases with increasing length of the fibers of these brushes. Reaction forces between the fibers of closed brushes are thus responsible for the second peak of the volume of water that is captured by model brushes.

Indeed, a peak that may correspond to the peak in the closed state has been measured in recent experiments that use brushes, wherein fibers are end-grafted in the radial directions of the substrates. ${ }^{3}$ Our theory predicts that such brushes also show a peak in the closed state, analogously to the present model brushes, where their fibers are endgrafted in the normal to the substrate surface; see Supplementary Figure S5a. For cases in which the radius of the brush substrate is smaller than the gravito-elastic length, $d<\lambda_{g}$, in the hydrostatic regime, the only relevant length scale is the gravito-elastic length $\lambda_{g}$. In these cases, the volume that is rescaled by $\pi L \lambda_{g}{ }^{2}$ is a function only of a fiber length that is rescaled by gravito-elastic length $\lambda_{g}$; with this rescaling, the volume of water that is measured for different numbers of fibers (approximately) collapses to one curve (see Figure 4c). Our theory predicts the volume of water in reasonable agreement with experiments; see Figure 4c.

\section{DISCUSSION}

Our theory predicts that model brushes suppress droplets with a physical mechanism that is significantly different from that of a single conical fiber. A single conical fiber stabilizes droplets by capillary forces that are generated on the surface of droplets owing to the 
varying cross-section of conical fibers. However, these capillary forces do not operate with water in brushes because the water fills the entire space between the fibers of the brushes; the surface energy at the fiberliquid interface is constant. Indeed, the volume of unwanted droplets is limited by radius $\rho_{L}$ of the brush ends because these droplets result from the rupture of capillary bridges; model brushes eliminate remnant droplets by closing their ends. This is a collective behavior of many flexible fibers, which is considerably different from that of a single fiber wetted by a droplet. The elastic deformations of wetted flexible materials have been treated in recent studies. ${ }^{3,4,7-11}$ In contrast to these studies, our theory emphasizes the effect of closing the ends of brushes on the hydrostatic properties that are relevant to the function of brushes as liquid-transfer systems.

Our theory makes a few experimentally accessible predictions: First, in the hydrostatic regime, $d>\lambda_{c}^{2} / \lambda_{g}$, the closing length, $L_{\text {clo }}\left(\sim \lambda_{g}\right)$, of model brushes is independent of radius $d$ of their substrates, in contrast to brushes of flexible plates because the local bending stiffness of the ambient-water interfaces of model brushes is inversely proportional to distance $\rho(z)$ between the interface and the central axis of the brush; see Figure 1 . The closing length, $L_{\text {clo }}$, of model brushes is relatively sensitive to radius $a$ of the cross-section of their fibers; see equation (5) (because the bending rigidity of fibers scales with $\left.k_{\mathrm{fib}} \sim a^{4}\right) .{ }^{16,20}$ Second, the volume of water that is captured by model brushes is a non-monotonic function of the length of their fibers, with one peak in the open state and another peak in the closed state. The peak in the closed state has been measured in recent experiments; ${ }^{3}$ however, the peak in the open state has not. With appropriate rescaling, the volume of water that is measured in various experimental conditions will collapse to one master curve as long as the system is in the same regime; in the hydrostatic regime, the volume of water should be rescaled by $\pi d^{2} L$, and the fiber length should be rescaled by gravito-elastic length $\lambda_{g}$.

Our theory is relatively generic, but it is ideally tested by experiments that satisfy the following conditions: First, flexible fibers are end-grafted in the normal to the substrate surface. The scaling relationships predicted by our theory may not be effective for model brushes, where their fibers are end-grafted in the radial direction of substrates. When the radius of the substrates of these brushes is smaller than the gravito-elastic length, $d<\lambda_{g}$, the radius $d$ is no longer a relevant length scale, and the volume of water that is captured by these brushes scales with $\pi \lambda_{g}^{2} L$; see Figure 4 c. Second, the number of fibers of a model brush is large enough so that the brush is approximately treated as a cylindrically symmetric system. Third, model brushes are removed from a water bath very slowly. When brushes are removed with finite speeds, this drives hydrodynamic flows in capillary bridges, and thus, the volume of water that is included in model brushes from the capillary bridges may be sensitive to the speeds of the brushes. We use a simple (approximate) treatment whereby capillary bridges rupture at the positions of the minimal cross-sections because this minimizes the surface energy. It is not obvious whether our results are indeed an asymptotic solution for a brush speed of zero; however, we expect that this treatment is a sufficiently good approximation for the scope of this paper.

Chinese calligraphic brushes, where fibers are end-grafted to the central regions (mantle) and the edges (outer layer) of their substrates ${ }^{21}$ may be treated in an extension of our theory. The conical ends of the fibers of the mantle do not generate capillary forces because the surface energy at fiber-water interfaces is constant as long as the space between the fibers is filled with water. If the mantle fibers are relatively long, the mantle may limit the elastic deformation of the outmost fibers owing to the excluded volume interactions between them. In contrast, when the fibers in the outer layer are longer than the fibers in the mantle, the ends of Chinese brushes are closed by fibers in the outer layer, just as the fibers of our model brushes are. The conical ends of the fibers and the length of fibers in the outer layer, relative to the fibers in the mantle, thus have an important role in suppressing unexpected drops of ink. ${ }^{1}$ These results have not been predicted by phenomenological models that do not treat interfacial forces. ${ }^{21,22}$

Our theory predicts that model brushes eliminate unwanted droplets by closing their ends. Moreover, this theory predicts that the volume of water that is captured by these brushes is a non-monotonic function of the length of their fibers, with one peak in the open state and the other peak in the closed state. These results are explained by the fact the area of the ends of closed brushes is zero, and the fibers of closed brushes show excluded volume interactions. Our theory demonstrates that closing the ends of Chinese calligraphic brushes significantly affects the hydrostatic properties of these brushes. Our theory may provide insight into the physics of the wetting of other fibrous systems ${ }^{23-26}$ that show interactions between flexible fibers. Moreover, this theory may guide the designs of bio-inspired liquid-transfer devices of flexible fibers to suppress unexpected drop of liquids.

\section{CONFLICT OF INTEREST}

The authors declare no conflict of interest.

\section{ACKNOWLEDGEMENTS}

We thank the National Research Fund for Fundamental Key Projects (2013CB933000), the National Natural Science Foundation of China (21574005), the Program for New Century Excellent Talents in Universities (NCET-13-0024), and Fundamental Research Funds for the Central Universities for their financial support. MD is supported by the Chinese Central Government in the 'Thousand Talents' program and an NSFC grant (21434001).

Author contributions: TY and MD performed the theoretical part of the research, whereas QM, QW, HL and LJ performed the experimental part of the research. All authors designed the research and wrote the paper. The main idea of this research was created by assembling the ideas of all authors.

1 Wang, Q., Su, B., Liu, H. \& Jiang, L. Chinese brushes: controllable liquid transfer in ratchet conical hairs. Adv. Matter 26, 4889-4894 (2014).

2 Ju, J., Bai, H., Zheng, Y., Zhao, T., Fang, R. \& Jiang, L. A multi-structural and multi-functional integrated fog collection system in cactus. Nat. Commun. 3, 1247 (2012).

3 Meng, Q., Wang, Q., Liu, H. \& Jiang, L. A bio-inspired flexible fiber array with an open radial geometry for high efficient liquid transfer. NPG Asia Mater. 6, e125 (2014).

4 Reis, P. M., Hure, J., Jung, S., Bush, J. W. M. \& Clanet, C. Grabbing water. Soft Matter 6, 5705-5708 (2010).

5 Lorenceau, É. \& Quéré, D. Drops on a conical wire. J. Fluid Mech. 510, 29-45 (2004).

6 de Gennes, P. G., Brochard-Wyart, F. \& Quéré, D. Capillarity and Wetting Phenomena (Springer-Verlag, New York, NY, USA, 2004).

7 Duprat, C., Protière, S., Beebe, A. Y. \& Stone, H. A. Wetting of flexible fibre arrays. Nature 482, 510-513 (2012).

8 Bico, J., Roman, B., Moulin, L. \& Boudaoud, A. Elastocapillary coalescence in wet hair. Nature 432, 690 (2004).

9 Py, C., Bastien, R., Bico, J., Roman, B. \& Boudaoud, A. 3D aggregation of wet fibers. Europhys. Lett. 77, 44005 (2007).

10 Roman, B. \& Bico, J. Elasto-capillarity: deforming an elastic structure with a liquid droplet. J. Phys. Condens. Matter 22, 493101 (2010).

11 Rivetti, M. \& Antkowiak, A. Elasto-capillary meniscus: pulling out a soft strip sticking to a liquid surface. Soft Matter 9, 6226-6234 (2013).

12 Chiodi, F., Roman, B. \& Bico, J. Piercing an interface with a brush. Europhys. Lett. 90 44006 (2010).

13 Ou-Yang, Z. C. \& Helfrich, W. Instability and deformation of spherical vesicles by pressure. Phys. Rev. Lett. 59, 2486-2488 (1987). 
14 Ou-Yang, Z. C., Liu, J. X. \& Xie, Y. Z. Geometric Methods in the Elastic Theory of Membranes in Liquid Crystal Phases (World Scientific: Singapore, 1999).

15 Ou-Yang, Z. C. \& Helfrich, W. Bending energy of vesicle membranes: general expression for the first, second, and third variation of the shape energy and applications to spheres and cylinders. Phys. Rev. A. 39, 5280-5288 (1989).

16 Landau, L. D. \& Lifshitz, E. M. Theory of Elasticity 3 edn (Elsevier, Oxford, UK, 1986).

17 Padday, J. F. \& Freud, H. Z. A theory of the ring method for the determination of surface tension. J. Am. Chem. Soc. 52, 1772-1782 (1930).

18 Freud, B. B., Pitt, A. R. \& Pashley, R. M. Menisci at a free liquid surface: surface tension from the maximum pull on a rod. J. Chem. Soc. 75, 2827-2838 (1975).

19 Debregeas, G. \& Brochard, F. Nucleation radius and growth of a liquid meniscus. J. Colloid. Interface Sci. 190, 134-141 (1997).

20 Audoly, B. \& Pomeau, Y. Elasticity and Geometry: From Hair Curls to the Nonlinear Response of Shells (Oxford University Press, Oxford, UK, 2010).

21 Chu, N. S. H. \& Tai, C. L. Real-time painting with an expressive virtual Chinese brush. IEEE Comput. Graph 24, 76-85 (2004).

22 Bai, B., Wong, K. W. \& Zhang, Y. in An Efficient Physically-Based Model for Chinese Brush. Frontiers in Algorithmics, Lecture Notes in Computer Sciences, Vol. 4613 (eds Preparata F. P. \& Fang Q.) 261-270 (Springer, Berlin, Heidelberg, Germany, 2007).

23 Beckrich, P., Weick, G., Marques, C. \& Charitat, T. Compression modulus of macroscopic fiber bundles. Euro. Phys. Lett. 64, 647-653 (2003).
24 Goldstein, R. E., Warren, P. B. \& Ball, R. C. Shape of a ponytail and the statistical physics of hair fiber bundles. Phys. Rev. Lett. 108, 078101 (2012).

25 Papadopoulos, P., Deng, X., Mammen, L., Drotlef, D. M., Battagliarin, G., Li, C., Müllen, K., Landfester, K., del Campo, A., Butt., H. J. \& Vollmer, D. Langmuir 28, 8392-8398 (2012).

26 Pilat, D. W., Papadopoulos, P., Schäffel, D., Vollmer, D., Berger, R. \& Butt, H. J. Dynamic measurement of the force required to move a liquid drop on a solid surface. Langmuir 28, 16812-16820 (2012).

This work is licensed under a Creative Commons Attribution 4.0 International License. The images or other third party material in this article are included in the article's Creative Commons license, unless indicated otherwise in the credit line; if the material is not included under the Creative Commons license, users will need to obtain permission from the license holder to reproduce the material. To view a copy of this license, visit http:// creativecommons.org/licenses/by/4.0/

Supplementary Information accompanies the paper on the NPG Asia Materials website (http://www.nature.com/am) 